\title{
EL DERECHO INTERNACIONAL DEL MEDIO AMBIENTE Y SU RELACIÓN CON OTRAS RAMAS DEL DERECHO INTERNACIONAL*
}

DOI: http://dx.doi.org/10.17981/juridcuc.11.1.2015.13

Recibido: 26 de Mayo de 2015 / Revisado: 24 de Julio de 2015 / Aceptado: 27 de Julio de 2015

\author{
Martin Canepa ${ }^{* * *}$ \\ Universidad de Buenos Aires (UBA)
}

Puede citar el presente artículo así: / To reference this article:

Canepa, M. (2015). El derecho Internacional del Medio Ambiente y su relación con otras ramas del Derecho Internacional. Jurídicas CUC, 11(1), 293-310. doi: http://dx.doi.org/10.17981/juridcuc.11.1.2015.13

\section{Resumen}

La protección del medio ambiente se ha constituido en uno de los temas principales de discusión a nivel internacional en distintos foros y organizaciones internacionales. El análisis de la problemáticas que rodean al medio ambiente como la contaminación, la protección de la flora y la fauna, del agujero de ozono entre otros, son temas que preocupan a la comunidad internacional entera. El Derecho internacional del medio ambiente procura regular las relaciones que se dan entre los Estados y entre éstos y las organizaciones internacionales en materia ambiental. El estudio de la relación con las otras ramas del Derecho internacional como el Derecho internacional de los derechos humanos, el Derecho humanitario y el Derecho internacional económico permite demostrar el interés actual de la comunidad internacional por los temas que conciernen al medio ambiente.

\section{Palabras clave:}

Derecho internacional del medio ambiente, protección, Derecho internacional económico, Derecho internacional de los derechos humanos, Derecho internacional humanitario

\footnotetext{
" Articulo de reflexión realizado con recursos propios del autor.

** Magister en Relaciones Internacionales, Abogado, Facultad de Derecho, Universidad de Buenos Aires. Correo Electrónico: martincanepa@derecho.uba.ar
} 


\title{
THE INTERNATIONAL ENVIRONMENTAL LAW AND ITS RELATIONSHIP WITH OTHER BRANCHES ON INTERNATIONAL LAW
}

\begin{abstract}
Protecting the environment has become one of the main topics of discussion around the world at various international conferences and organizations. The analysis of the issues regarding the environment such as pollution, protection of flora and fauna, and the detriment of the ozone layer, among other subjects, concerns the entire international community. International Environmental Law seeks to regulate relations between States, as well as between them and international environmental organizations. The study of the relationship with other branches of International Law such as International Human Rights Law, International Humanitarian Law and International Economic Law demonstrate the current interest of the international community on environmental topics.
\end{abstract}

Keywords:

International Environmental Law, Protection, International Economic Law, International Human Rights Law, International Humanitarian Law 


\section{INTRODUCCIÓN}

El Derecho internacional del medio ambiente ha experimentado en los últimos años un gran desarrollo por medio del cual ha logrado consolidar la estructura de sus principios fundamentales y las bases de sus conceptos teóricos que constituyen la base del mismo. El Derecho internacional público hace ya cierto tiempo se ha visto fragmentado cada vez más en distintas ramas de conocimiento que estudian los temas de interés relativos al derecho internacional. Como consecuencia según Abi-Saab (1999), esta fragmentación también ha tenido lugar en la creación de distintos tribunales, ya sea de tipo judicial o arbitral, que se encargan de resolver las controversias que surjan de los diversos campos de estudio (Romano, 2011). En este sentido, se ha creado el Tribunal del Derecho del Mar, la Corte Penal Internacional y la Corte Europea de Derechos Humanos, entre otros. Todavía no se ha establecido un tribunal específico sobre Derecho internacional del medio ambiente, sino que los conflictos que sobrevinieren en este sentido son resueltos por los tribunales ya existentes que tienen competencia para ciertas materias puntuales o para temas de Derecho internacional general, tal como la Corte Internacional de Justicia, la cual tiene competencia para entender en cualquier asunto que verse sobre el Derecho internacional general.

El objetivo de este trabajo será el análisis de la relación existente entre el Derecho internacional del medio ambiente y otras ramas del Derecho internacional. En primer lugar, intentaremos dar un concepto de esta rama del derecho para luego concentrarnos en su relación con las siguientes ramas: Derecho internacional económico, Derecho internacional de los derechos humanos y Derecho internacional humanitario. De esta forma, trataremos de vislumbrar cuál es la naturaleza, alcance y extensión de la relación entre estas ramas y el derecho internacional del medio ambiente.

\section{Concepto}

El Derecho internacional del medio ambiente es aquella rama del Derecho internacional público que se ocupa de regular los aspectos que 
hacen a la protección del medio ambiente a partir de la relación entre los Estados en el marco de sus relaciones internacionales. Esta rama del derecho se perfila como una rama autónoma, no solo en el ámbito del Derecho internacional público sino del Derecho general. (Drnas 2009).

\section{Derecho internacional económico y Derecho internacional del medio ambiente}

La relación entre el Derecho internacional económico y el Derecho internacional del medio ambiente muestra quizás una de las problemáticas cada vez más presentes en el mundo del comercio internacional y las inversiones extranjeras en terceros Estados. Con la profundización de la globalización y el comercio entre Estados, se han firmado una gran cantidad de tratados internacionales tanto a nivel multilateral y bilateral como regional, en este último caso dentro del marco de sistemas de integración, los cuales prevén en algunos supuestos sanciones para casos de contaminación ambiental como consecuencia de una inversión o resultado de alguna explotación industrial, y en otros casos, si bien no se prevé expresamente una solución para estos supuestos, fue a través del sistema de solución de controversias creado bajo el auspicio de estos tratados que se logró dar una solución a los daños ambientales producidos. A modo de presentar cómo se ha hecho efectivo el control sobre daños producidos al medio ambiente en relación con actividades comerciales entre Estados es interesante destacar ciertos casos jurisprudenciales que ilustran con claridad estos supuestos.

Dentro del campo de los casos sometidos al CIADI, encontramos varios laudos de tribunales arbitrales en donde la cuestión ambiental se puso de relieve como contracara en casos de inversiones extranjeras.

En los caso contra Costa Rica (Ciadi N ${ }^{0}$ Arb/08/1 y Ciadi N ${ }^{o}$ Arb/09/20, 2012), en donde la parte inversora demandaba al Estado por expropiación, el Tribunal determinó que se produjo una expropiación de facto y que se trató de una expropiación ilegal ya que no había existido una compensación a causa de las medidas que produjeron el menoscabo de los derechos de propiedad de la parte demandante y que 
consistían en medidas equivalentes a una expropiación; en este caso, la conversión de una determinada zona en reserva nacional. En consecuencia, la decisión de crear una reserva que se superponía en parte con la propiedad del complejo hotelero consistió en una medida equivalente a la expropiación que no fue objeto de compensación y, que por lo tanto, debe ser indemnizada. A pesar de que el fin último del Estado era dar protección a una comunidad de tortugas marinas, el Tribunal consideró que igualmente se había violado el derecho del inversor ya que la medida se traducía en una expropiación.

Dentro del ámbito de la OMC debemos mencionar el artículo XX del GATT, cuyo inciso $b$ establece como excepción a uno o varios principios generales del comercio, el hecho de que las medidas adoptadas sean "necesarias para proteger la salud y la vida de las personas y de los animales o para preservar los vegetales", y también el inciso $g$ del mismo artículo que ampara las medidas que se adopten cuando el fin consista en la conservación de los recursos naturales agotables.

En el Caso EEUU-Gasolina(1987), el órgano de apelación estableció que para que la primera excepción anteriormente mencionada proceda es necesario que la política por la que se toma la medida esté destinada a proteger la salud y la vida de las personas y de los animales o a preservar los vegetales.

En relación al segundo supuesto, el órgano de apelación en el Caso EEUU-Camarones (1998), dispuso que "el término genérico recursos naturales del párrafo g) del artículo XX no es estático en su contenido o en sus referencias sino más bien por definición, evolutivo. Por consiguiente, es oportuno advertir que las modernas convenciones y declaraciones internacionales hacen frecuentes referencias a los recursos naturales incluyendo dentro de los mismos tanto a los recursos vivos como a los no vivos".

Resulta interesante destacar que el sistema de solución de controversias de la OMC es un sistema híbrido ya que se trata de paneles constituidos ad hoc y de un órgano de apelación con carácter permanente que ha sabido desarrollar una jurisprudencia constante a través de los años y en donde las cuestiones ambientales se han suscitado con cada vez más frecuencia como argumento por parte de los Estados para 
justificar el no cumplimiento con de alguno de los principios generales al comercio y, en consecuencia, para poder ampararse en alguna de las excepciones al comercio que el propio GATT establece. (Delich, 2011).

En cuanto a la labor de la Corte Internacional de Justicia podemos mencionar el caso sobre la caza de ballenas (arrêt du 31 mars 2014), en esta oportunidad el juez Cançado Trindade en su opinión individual sostiene que los principios de prevención y precaución están estrechamente ligados en el presente caso y deben inspirar y dar forma a todo programa llevado a cabo a título de un permiso especial, en donde la caza se encuentra limitada en virtud del artículo VIII de la Convención para la regulación de la caza de la ballena (Convención Ballenera, 1946). Concluye sobre este punto que:

dans le domaine du droit international de l'environnement d'une manière générale, et en ce qui concerne la convention en particulier, la conservation des ressources marines vivantes est devenue, au fil du temps, un intérêt commun prévalant sur la rentabilité commerciale recherchée unilatéralement par les Etats

Otro caso que resulta interesante señalar es el de las plantas de celulosa sobre el río Uruguay, en donde la Corte Internacional de Justicia (Judgment, 20/04/2010) sostuvo que Uruguay había violado las obligaciones procedimentales que establecía el Estatuto del Río Uruguay de 1975 en los artículos 7 a 12, no así las obligaciones sustantivas. La consecuencia jurídica de este incumplimiento consistió en la reparación por medio de satisfacción, dada a través de la declaración de la misma Corte en relación con la violación llevada a cabo por el Gobierno Uruguayo (Gonzalez, 2010). La posición argentina sostenía que las plantas de pasta celulosa eran contaminantes, sin embargo, la Corte concluyó que hasta el momento no se había podido probar que las plantas contaminaban o podían llegar a contaminar. En este sentido, la preocupación de la Argentina pone de relieve la idea del desarrollo sostenible. Como sostiene Gutiérrez (2010, p. 4):

El derecho al desarrollo es un derecho de todo Estado pero necesariamente deben encontrarse vías adecuadas para que sea sostenible, respetando el derecho de las generaciones presentes y venideras a un medio ambiente sano. 


\section{Derecho internacional de los derechos humanos y el Derecho internacional ambiental}

El Derecho internacional ambiental y el Derecho internacional de los derechos humanos de manera progresiva se han venido complementando y han trabajado de manera más o menos conjunta en el sentido de brindar protección a los particulares frente a casos de contaminación, deforestación u otros tipos de daños ambientales. Tradicionalmente el contenido de los derechos humanos no incluía el derecho a un medio ambiente sano, pero con el tiempo, fue evolucionando y algunos Tratados de Derechos Humanos han incorporado dentro de su catálogo el derecho a un ambiente sano junto con la necesidad de que los Estados se encarguen de realizar un desarrollo sustentable que permita la conservación de los recursos naturales presentes para el uso y aprovechamiento de los mismos por las generaciones futuras. A su vez, muchos Estados han incorporado, ya sea por medio de una ley o en sus disposiciones constitucionales relativas a los derechos fundamentales, el derecho a un medio ambiente sano como un derecho básico y elemental, cuya protección resulta necesaria.

Un ejemplo de cómo algunos Estados han receptado la protección del derecho al medio ambiente es el artículo 41 de la Constitución argentina luego de la reforma de 1994, el cual dispone:

Todos los habitantes gozan del derecho a un ambiente sano, equilibrado, apto para el desarrollo humano y para que las actividades productivas satisfagan las necesidades presentes sin comprometer las de las generaciones futuras; y tienen el deber de preservarlo. El daño ambiental generará prioritariamente la obligación de recomponer, según lo establezca la ley.

Las autoridades proveerán a la protección de este derecho, a la utilización racional de los recursos naturales, a la preservación del patrimonio natural y cultural y de la diversidad biológica, y a la información y educación ambientales. (1994).

La relación entre Derechos Humanos y el Derecho ambiental se presenta de una manera sumamente estrecha, ya que los actos que van en 
contra del medio ambiente y que pueden causar la destrucción de ecosistemas como la desertificación, tala indiscriminada o contaminación del agua traen como consecuencia abusos en el ámbito de los derechos humanos de las personas afectadas por este accionar destructivo.

De esta manera han surgido coaliciones medioambientales de derechos humanos en los últimos años cuyo trabajo se concentra en distintos ejes como los ríos, las costas playeras o los bosques. La sinergia entre Derechos humanos y Derecho ambiental está sucediendo a través del trabajo de organizaciones no gubernamentales y el activismo transnacional. (Conca, 2005)

Un gran sector de la doctrina se ha venido pronunciando acerca de la inclusión del derecho humano al agua como un derecho incluido en el Pacto de derechos económicos, sociales y culturales. En este sentido, el Comité de derechos económicos, sociales y culturales en su observación $\mathrm{n}^{\circ} 15$ (2003), estableció que los Estados deben velar porque los terceros que estuvieren a cargo de la administración o suministro de los servicios de agua otorguen un acceso físico al agua, a un precio razonable, sin discriminación, en condiciones de salubridad y calidad aceptables y cantidad suficiente. El Comité, en la misma observación consideró que el derecho humano al agua se encuentra implícitamente contenido en los artículos 11 y 12 del Pacto de derechos económicos, sociales y culturales, y explícitamente, en el artículo 14(2) de la Convención sobre la eliminación de todas las formas de discriminación contra la mujer y en el artículo 24(2) de la Convención relativa a los derechos del niño (Peterson, 2009, p. 26).

Como establece Bansal (2011, p. 128):

Environmental policy is designed in part to preserve the environment for future generations. This is because future generations are not present during the creation of international agreements; members of the current generation must represent their interests.

En el caso Trail Smelter Arbitration (1938) se pone de manifiesto la responsabilidad de los Estados por casos de contaminación o daños al medio ambiente en el territorio de otro Estado, el Tribunal arbitral expresó que: 
Under the principles of international law, ... no State has the right to use or permit the use of its territory in such a manner as to cause [environmental] injury ... in or to the territory of another or the properties of persons therein, when the case is of serious consequence and the injury is established by clear and convincing evidence.

En el ámbito africano es importante destacar dos instrumentos. Por un lado, la African Convention on Nature and Natural Resources cuyo artículo 3 establece un deber de la Estados para asegurar el derecho al medio ambiente y el desarrollo sustentable. A su vez, la Convención Africana sobre derechos humanos y de los pueblos dispone en su artículo 24 un derecho para los pueblos a: "General satisfactory environment favourable to their development."

Algunos Estados africanos, por ejemplo Kenia (1999), han establecido el derecho universal a un ambiente sano y limpio para cualquier residente en el territorio de dicho Estado. A su vez, la Carta de derechos contenida en la Constitución de 2010 también establece el derecho a un ambiente sano y limpio transformando el derecho al medio ambiente en un derecho básico en el sistema legal keniano (Kibugi, 2011, p. 169).

Como establece Du Pleiss (2011, p. 39) en relación con los países del África:

The duties of African state governments in terms of the environmental right are accordingly not limited to natural resource protection but extend to issues related to a 'general satisfactory environment' such as health, well-being and livelihood.

Esta última afirmación nos lleva a considerar el derecho a un ambiente sano como un derecho humano fundamental que va de la mano con otros derechos como el derecho a la vida, la salud y la vivienda, entre otros.

En el ámbito europeo, la Carta de los Derechos Fundamentales de la Unión Europea (2010) en su artículo 37 sobre la protección del medio ambiente establece:

En las políticas de la Unión se integrarán y garantizarán, conforme al principio de desarrollo sostenible, un nivel elevado de protección del medio ambiente y la mejora de su calidad. 
Esta disposición corresponde al ámbito legal de la Unión Europea. Los Estados que son parte del Consejo de Europa no han establecido expresamente un derecho a un ambiente sano y limpio, sin embargo, la Corte Europea de Derechos Humanos ha logrado por vía indirecta (Miranda, 2007, p. 75-93), teniendo en cuenta la mencionada ausencia de una disposición expresa en la Convención Europea de Derechos Humanos, tutelar el derecho a un ambiente sano, por medio de una interpretación progresiva del artículo 8 de dicha Convención, el cual protege la vida privada y familiar, el domicilio y la correspondencia bajo los siguientes términos:

1. Toda persona tiene derecho al respeto de su vida privada y familiar, de su domicilio y de su correspondencia.

2. No podrá haber injerencia de la autoridad pública en el ejercicio de este derecho sino en tanto y en cuanto esta injerencia esté prevista por la ley y constituya una medida que en una sociedad democrática, sea necesaria para la seguridad nacional, la seguridad pública, el bienestar económico del país, la defensa del orden y la prevención de las infracciones penales, la protección de la salud o de la moral, o la protección de los derechos y las libertades de los demás.

En el caso López Ostra v. España (Sentencia A/303-C de 1994), la Corte Europea de Derechos Humanos se pronunció por primera vez acerca del principio en virtud del cual los daños ambientales ocasionados a la colectividad, aun cuando no fueran considerados graves de manera tal de poner en peligro la vida de los individuos, pueden de todas formas perjudicar su bienestar y privarlo del disfrute del derecho a su domicilio, causar daños a la vida privada y familiar y a la salud.

\section{Derecho internacional humanitario y Derecho internacional ambiental}

El Derecho internacional humanitario (Bothe, 2010), regula el derecho aplicable en casos de conflictos armados, ya sean de carácter internacional o no internacional. Siguiendo el razonamiento de Gutiérrez (2014) podemos definirlo como: "el conjunto de normas jurídicas de fuente convencional y consuetudinaria que tiene por objeto proteger a las víctimas de los conflictos armados a la par que poner límites a los métodos y medios de combate (...)" (2014, p. 16). 
Los conflictos armados pueden provocar serios daños al medio ambiente, directos e indirectos, los cuales a su vez traen consecuencias peligrosas para la salud, la flora, la fauna y la seguridad de las personas, de manera tal que estos riesgos se vean reducidos el Derecho internacional de los conflictos armados ha intentado dar una protección adecuada al medio ambiente en situaciones de conflicto armado.

Uno de los primeros intentos de dar protección al medio ambiente durante conflictos armados tuvo lugar en la década de 1970 como consecuencia de los acontecimientos que tuvieron lugar a raíz de la guerra de Vietnam. Nuevamente, entre 1990-1991 luego de los derrames de petróleo provocados durante la guerra del Golfo, el interés volvió a resurgir.

En 1976, la Comisión de la Conferencia de Desarme (CCD) aprobó la Convención sobre la prohibición de utilizar técnicas de modificación ambiental con fines militares u otros fines hostiles (ENMOD, 1976). El tratado regula el uso de técnicas de modificación ambiental como medio para causar daño al enemigo. La ENMOD prohíbe expresamente "las técnicas de modificación ambiental que tengan efectos extensos, duraderos o graves como medios para producir destrucciones".

Siguiendo esta línea, la resolución 47/37 de la Asamblea General de la ONU (1993), insta a los Estados a adoptar medidas para cumplir con el Derecho internacional ambiental durante los conflictos armados. El Protocolo adicional I a los Convenios de Ginebra de (1949) establece una especie de umbral de inadmisibilidad de daño ambiental, considerando tal, a aquel que sea extenso, duradero y grave. Parecería ser que los conflictos armados de carácter no internacional no estarían cubiertos por el Derecho internacional humanitario en lo que respecta a la protección del medio ambiente, lo cual generaría graves problemas ya que hoy en día la mayoría de los conflictos armados no son de carácter internacional.

A su vez, el artículo 55 del Protocolo adicional I establece:

En la realización de la guerra se velará por la protección del medio ambiente natural contra daños extensos, duraderos y graves. Esta protección incluye la prohibición de emplear métodos o medios de hacer la guerra que hayan sido concebidos para causar o de los que quepa prever que causen tales daños al medio ambiente natural, comprometiendo así la salud o la supervivencia de la población. 
Una de las cuestiones problemáticas que se plantean, es el hecho de que existe una alta probabilidad de que los elementos del medio ambiente se conviertan en objetivos militares, lo que llevaría a invalidar su protección. La posibilidad de aplicar las normas del Derecho internacional ambiental a situaciones de conflictos armados va a depender de las disposiciones contenidas en los acuerdos multilaterales sobre Derecho ambiental. En este sentido algunos acuerdos determinan que serán aplicables directa o indirectamente durante situaciones de conflicto armado, otros específicamente establecen su inaplicabilidad y en la mayoría de los casos no se hace mención al tema, lo que genera mayor incertidumbre al respecto.

Las declaraciones, en materia de Derecho internacional ambiental resultan de gran relevancia, ya que a través de ellas, se han asentado muchos de sus principios fundamentales. La Declaración de la Conferencia de las Naciones Unidas sobre el Medio Humano (1972) establece en el principio 21 que: "los Estados tienen [...] la obligación de asegurar que las actividades que se lleven a cabo dentro de su jurisdicción o bajo su control no perjudiquen al medio de otros Estados o de zonas situadas fuera de toda jurisdicción nacional". Este principio se relaciona íntimamente con la posibilidad de aplicar las normas del Derecho internacional ambiental durante la vigencia de un conflicto armado.

Por su parte, la Declaración de la Conferencia de las Naciones Unidas sobre el medio ambiente y el desarrollo (1992) en el principio 24 establece: "[l]a guerra es, por definición, enemiga del desarrollo sostenible. En consecuencia, los Estados deberán respetar las disposiciones del derecho internacional que protegen al medio ambiente en épocas de conflicto armado y cooperar en su ulterior desarrollo, según sea necesario"

En este sentido, la Carta Mundial de la Naturaleza (1982) determina en su principio 5 que: "se protegerá a la naturaleza de la destrucción que causan las guerras u otros actos de hostilidad".

La Corte Internacional de Justicia en la opinión consultiva acerca del empleo de armas nucleares (1996) establece que: 
La Cour constate ainsi que, si le droit international existant relatif à la protection et à la sauvegarde de l'environnement n'interdit pas spécifiquement l'emploi d'armes nucléaires, il met en avant d'importantes

considérations d'ordre écologique qui doivent être dûment prises en compte dans le cadre de la mise en œuvre des principes et règles du droit applicables dans les conflits armés.

De esta manera, hace específicamente referencia a la protección del medio ambiente y a la consideración de este aspecto en la ejecución de los principios aplicables a los conflictos armados.

En relación a la responsabilidad del invidivuo dentro del ámbito del Derecho penal internacional, debemos hacer referencia al Estatuto de Roma, cuyo artículo 8 relativo a los crímenes de guerra, en el inciso 2.b.iv considera que a los efectos del Estatuto, también se entiende por crimenes de guerra, otras violaciones graves de las leyes y usos aplicable en los conflictos armados internacionales dentro del marco establecido por el Derecho internacional, entre las cuales menciona:

Lanzar un ataque intencionalmente, a sabiendas de que causará pérdidas incidentales de vidas, lesiones a civiles o daños a bienes de carácter civil o daños extensos, duraderos y graves al medio ambiente natural que serían manifiestamente excesivos en relación con la ventaja militar concreta y directa de conjunto que se prevea.

En el Manual de San Remo sobre Derecho internacional aplicable a los conflictos armados en el mar (1994) se afirma que: "[l]os métodos o medios de guerra deben emplearse con la debida consideración por el medio ambiente natural". Asimismo, de acuerdo con el artículo 44 del Manual: "quedan prohibidos los daños y las destrucciones del medio ambiente natural no justificados por las necesidades militares y que se causen arbitrariamente".

La doctrina, como podemos observar, también se ha hecho eco de los estragos que producen los conflictos armados en el medio ambiente y que necesitan ser estudiados y considerados por los Estados para poder llevar a cabo tareas de prevención al momento de dirigir un combate y elegir los medios y métodos aplicables al mismo. 


\section{CONCLUSIÓN}

Luego de haber analizado la relación del Derecho internacional ambiental con otras ramas específicas del Derecho internacional público podemos observar que las características que presenta este vínculo poseen sus particularidades para cada uno de los casos.

En cuanto al Derecho Internacional de los Derechos Humanos, la relación existente se desenvuelve en el sentido de la inclusión o no del derecho a un ambiente sano como un derecho humano. A la luz de ciertos tratados internacionales y de la doctrina escogida, nos inclinaríamos por la respuesta afirmativa en el sentido de considerar que hoy en día, en virtud del desarrollo del Derecho internacional público, el derecho a un ambiente sano puede considerarse un derecho humano.

El Derecho internacional humanitario presenta otro tipo de relación con el Derecho internacional ambiental, la cual plantea que ciertos daños causados al medio ambiente pueden configurar un crimen de carácter internacional y también ser objeto de prohibición por parte de tratados internacionales específicos sobre la reglamentación de métodos de combate.

En lo que respecta al Derecho internacional económico, la relación con éste refleja la tensión existente entre las modernas relaciones comerciales actuales y la forma en que las inversiones extranjeras se desarrollan. La mayor parte de los casos en donde esta relación queda expuesta es en los supuestos de incumplimiento o conclusión de un contrato comercial. Muchas veces el Estado, receptor de la inversión o en cuyo territorio se lleva a cabo la relación comercial, manifiesta que la otra parte ha producido un daño al medio ambiente, y por lo tanto, la relación contractual no puede continuar. En tal caso, se impone una indemnización por los daños provocados o bien puede continuar pero tomando las medidas necesarias para hacer cesar los daños al medio ambiente y los perjuicios que éstos pueden producir a la población local.

Sin duda, el Derecho internacional ambiental se relaciona estrechamente con otras ramas del Derecho internacional con las cuales se complementa y a las cuales nutre de sus propios conceptos. La presencia de un Tribunal específico de solución de controversias sobre temas ambien- 
tales en el ámbito internacional no parece necesaria por el momento, ya que los tribunales existentes, tanto en el ámbito universal como regional, tienen competencia para casos que planteen cuestiones acerca de la violación a ciertas normas de derecho ambiental. Si bien en muchos casos la cuestión ambiental está conectada a otras ramas del Derecho, no resulta por eso menos efectiva la solución final que estos tribunales puedan ofrecer frente a un conflicto determinado.

\section{BIBLIOGRAFÍA}

Abi-Saab, G. (1999). Fragmentation or Unification: Some Concluding Remarks, New York University Journal of International Law and Politics, 31, 919-933.

Banco Mundial. Centro Internacional de arreglo de diferencias relativas a Inversiones. Caso CIADI No ARB/08/1. (16 de mayo de 2012).

Banco Mundial. Centro Internacional de arreglo de diferencias relativas a Inversiones. Caso CIADI N ARB/09/20. (16 de mayo de 2012).

Corte Europea de Derechos Humanos. Sentencia del 9 de diciembre de 1994. Caso López Ostra v. España. Serie A/303-C.

Cour Internationale de Justice. (Arrêt du 31 mars 2014). Chasse à la baleine dans l'Antarctique (Australie c. Japon). CIJ Recueil 2014.

Cour Internationale de Justice (Judgment 20 de abril de 2010). Case concerning Pulp Mills on the River Uruguay (Argentina v. Uruguay). CIJ Recueil 2010.

Cour Internationale de Justice (Avis consultatif du 8 juillet 1996). Licéité de la menace ou de l'emploi d'armes nucléaires. CIJ Recueil 1996.

Bansal, K. (2011). Transboundary environmental pollution: Understanding the intricacies of international law. Pretoria Student Law Review. Pretoria University Law Press (PULP). Johannesburg, (5), 128. Disponible en: http://www.pulp.up.ac.za/pdf/2013_02/2013_02.pdf

Bothe, M., Bruch, C., Diamond, J. \& Jensen, D. (2010). El derecho internacional y la protección del medio ambiente durante los conflictos armadas: lagunas y oportunidades. International Review of the Red Cross, (879).

Comité Internacional de la Cruz Roja. Protocolo adicional a los Convenios de Ginebra del (12 de agosto de 1949). Relativo a la protección de las vícti- 
mas de los conflictos armados internacionales (Protocolo I) del 8 de junio de 1977, 35(3). Recuperado de: https://www.icrc.org/spa/assets/files/other/ paiii-spanish-08.12.2005-clear19.12.pdf

Conca, K. Environmental Governance after Johannesburg: From Stalled Legalization to Environmental Human Rights?". Journal of International Law \& International Relations. Munk School/Faculty of Law, University of Toronto, 1(1-2): 131-133. Disponible en: http://www.jilir.org/docs/issues/ volume_1/1_9_CONCA_FINAL.pdf

Delich, V. (2011). Asimetrías, conflictos comerciales e instituciones internacionales. El caso del Mercosur en el sistema de solución de controversias de la OMC. Buenos Aires: Eudeba.

Drnas, Z. (2009). Fuentes del Derecho Medio Ambiente. Revista electrónica cordobesa de Derecho Internacional Público (RECorDIP), 1 (1), 2. Recuperado de: http://revistas.unc.edu.ar/index.php/recordip/article/view/37

Du Plessis, A. (2011). The balance of sustainability interests from the perspective of the African Charter on Human and Peoples' Rights. The balancing of interests in environmental law in Africa. Recuperado de: http://www. pulp.up.ac.za/pdf/2012_04/2012_04.pdf

González, S. (2010). Caso de las plantas de celulosa sobre el Río Uruguay: las consecuencias jurídicas del incumplimiento de Uruguay según la Corte Internacional de Justicia. eldial.com. Recuperado de: http://www.eldial. com/suplementos/internacional/internacional.asp

Gutiérrez, H. (2010). Una sentencia que ha de poner término a la controversia entre la Argentina y Uruguay. La ley. 7(1), 4.

Gutiérrez, H. (2014). Elementos de Derecho Internacional Humanitario. $1^{a}$ ed. Buenos Aires: Eudeba.

International Whaling Commision. Convenio Internacional para la regulación de la pesca de la ballena. (2 de diciembre de 1946). Recuperado de; https:// iwc.int/convention-es

Kibugi, R. (2011). Development and the balancing of interests in Kenya. The balancing of interests in environmental law in Africa. Pretoria University Law Press (PULP), Cape Town, p. 169. Disponible en: http://www.pulp. up.ac.za/pdf/2012_04/2012_04.pdf

Miranda, H. (2007). La protección del ambiente en el sistema europeo de Derechos Humanos. Panóptica, 1 (8), 75-93. 
Organización de las Naciones Unidas. (April 16, 1938). Trail Smelter Case US $v$ Canada. International Arbitral Awards 3. Recuperado de: http://legal. un.org/riaa/cases/vol_III/1905-1982.pdf

Organización de las Naciones Unidas. Comisión de la Conferencia de Desarme, CDD. (10 de diciembre de 1976). Convención sobre la prohibición de utilizar técnicas de modificación ambiental con fines militares u otros fines hostiles. ENMOD 1108. Serie de Tratados de las Naciones Unidas. Recuperado de: https://www.icrc.org/spa/resources/documents/misc/treaty-1976-enmod-convention-5tdm21.htm

Organización de Naciones Unidas. Comité de derechos económicos, sociales y culturales. (20 de enero de 2003). Observación general $n^{\circ} 15$. El derecho al agua (artículos 11 y 12 del Pacto Internacional de Derechos Económicos, Sociales y Culturales). Recuperado de: http://www.ciceana.org.mx/recursos/onu_derecho_agua.pdf

Organización de las Naciones Unidas. Declaración de la Conferencia de las Naciones Unidas sobre el Medio Humano. (16 de junio de 1972). Recuperado de: http://www.medioambiente.cu/declaracion_estocolmo_1972.htm

Organización de Naciones Unidas. (1992). Informe de la Conferencia de Naciones Unidas sobre Medio Ambiente y Desarrollo. Recuperado de: http:// www.unesco.org/education/nfsunesco/pdf/RIO_S.PDF

Organización Mundial del Comercio. (1987). Informe del Órgano de Apelación EEUU-Gasolina. Recuperado de: https://www.wto.org/spanish/tratop_s/ dispu_s/cases_s/ds2_s.htm

Organización Mundial del Comercio. (1998). Informe del Órgano de Apelación EEUU-Camarones. Recuperado de: https://www.wto.org/spanish/ tratop_s/dispu_s/cases_s/ds58_s.htm

Peterson, L. (2009). Droits humains et traités bilatéraux d'investissement, le rôle du droit relatif aux droits humains dans l'arbitrage des différends entre investisseur et États, Droits et Démocratie. Centre international des droits de la personne et du développement démocratique. 26.

República de Argentina. Senado de la Nación Argentina. (1994). Constitución argentina. Recuperado de: http://www.senado.gov.ar/deInteres

Repúblic of Kenia. Ministry of Environment and Natural Reosurces. (1999). Kenian Environmental Management and Coordination Act (EMCA) section 3. Recuperado de: http://faolex.fao.org/docs/pdf/ken41653.pdf 
Romano, C. (2011). A Taxonomy of International Rule of Law Institutions. Journal of International Dispute Settlement, 2 (1), 241-277. doi: 10.1093/ jnlids/idq024

Organización de Naciones Unidas. Resolución 47/37 de la Asamblea General de las Naciones Unidas, (9 de febrero de 1993), Protection of the Environment in Times of Armed Conflict, UN Doc. A/RES/47/37

Organización de Naciones Unidas. Resolución 37/7 de la Asamblea General de las Naciones Unidas, (28 de octubre de 1982), Carta Mundial de la Naturaleza.

Unión Europea. Consejo Europeo de Colonia. Carta de los Derechos Fundamentales de la Unión Europea (2010) Recuperado de: http://www.europarl.europa.eu/charter/pdf/text_es.pdf 